\title{
Rhinitis in Pregnancy among Saudi Women
}

Sameer Albahkaly ${ }^{1}$, Salwa Mohammed Bahkali ${ }^{2}$, Mohammed Ghazi Alotaibi ${ }^{3}$, Abdullah Mohammed Alghamdi ${ }^{4}$, Raseel Saleh Alswidan ${ }^{5}$, Maha Fahad bin Shafi ${ }^{6}$ and Sarah Suliman Almaiman ${ }^{7}$

1. Department of Ear, Nose \& Throat, King Abdulaziz. Medical City, Ministry of National Guard Health Affairs, Riyadh 11426, Saudi Arabia

2. Department of Family Medicine, King Abdulaziz Medical City, Ministry of National Guard Health Affairs (NGHA), Riyadh 11613, Saudi Arabia

3. Department of Ear, Nose \& Throat, King Salman Hospital, Minstry of Health, Riyadh 11757, Saudi Arabia

4. Department of Orthopedic, King Abdulaziz Medical City, Ministry of National Guard Health Affairs, Riyadh 11757, KSA

5. College of Medicine, King Saud University, Riyadh 12281, Saudi Arabia

6. College of Medicine, Taif University, Taif 21499, Saudi Arabia

7. Riyadh Colleges for Dentistry and Pharmacy, Riyadh 11613, Saudi Arabia

\begin{abstract}
OBJECTIVE: Rhinitis is common in Saud Arabia. Therefore, our study was designed to evaluate the prevalence, triggering factors, severity and progression of pregnancy rhinitis. METHODS: Prospective cross-sectional study was conducted in multiple medical centers in Riyadh, Saudi Arabia, during June and July 2014. It used an Arabic language self-administered questionnaire on a sample of 260 pregnant women. Random sampling was done on Saudi pregnant ladies whom visiting antenatal clinics in 8 governmental and private medical centers. RESULTS: The reported rhinitis during pregnancy in our study was $31.2 \%$ (CI $25.6 \%-37.2 \%$ ). Symptoms arising in first trimester appeared in $79.2 \%$ of pregnancy rhinitis (PR) cases and mostly go worsen. The most prevalent symptoms were nasal pruritus, followed by sneezing, congestion, and post nasal drip. The major triggering factor was dust, followed by Tobacco/Shisha smoke and perfume. Preexisting allergic diseases were markedly associated with developing pregnancy rhinitis. CONCLUSION: Rhinitis during pregnancy manifested in one third of Saudi pregnant ladies. Nasal pruritus was the most common symptom and dust was the widespread triggering factor.
\end{abstract}

Key words: Saudi, rhinitis, pregnancy, allergy, congestion, sneezing.

\section{Introduction}

Rhinitis is a widely common medical condition which is defined as inflammation of nasal mucosa, causing symptoms of runny, itchy, and obstructed nose with sneezing [1]. It is categorized broadly into allergic (AR) and non-allergic (NAR) types. AR is more common with a ratio of 2:1 [2]. Worldwide, allergic rhinitis prevalence ranges between $9 \%$ and $42 \%$ [3], which is higher in the developing countries and recently it has escalating rates [4]. Allergic disorders are common in KSA and allergic rhinitis was the highest observed subtype [5]. Rhinitis and AR

Corresponding author: Sameer Albahkaly, MD, FKSU, $\mathrm{AB}, \mathrm{SB}$, assistant professor, research field: otolaryngology. were as prevalent as $38.6 \%$ and $21.3 \%$, respectively.

Rhinitis presents during pregnancy as it does in non-pregnant state [6]. Pregnancy rhinitis (PR) refers to the occurrence of rhinitis and its symptoms during pregnancy [7], which typically decline within two weeks postnatal [8]. Despite its benign nature, it is one of the greatest bothering conditions affecting the quality of life for pregnant women, specially the AR type [9-11]. It is also a major concern facing doctors for its diagnosis and treatment $[12,13]$. Worldwide, $20 \%$ to $30 \%$ of women experience PR symptoms through their pregnancies $[10,14,15] . \mathrm{PR}$ is often underdiagnosed and yet being ignored in antenatal visits.

PR can present with AR or NAR, and symptoms of 
both types are hardly distinguished. The alterations of hormones through pregnancy, especially estrogen, beside the rise blood volume are considered to be the principal cause of PR. That results in direct or indirect increase in nasal vascular engorgement [10,14]. The main originator of allergic rhinitis resulting in its symptoms is the exposure to triggering allergens [16]. Furthermore, the precise reasons for developing PR have not yet been identified by researchers.

Although PR is a common and distressing condition to the mothers, no study has been conducted in Saudi Arabia in this regard. In order to establish a baseline data for respiratory health needs in antenatal programs in Saudi Arabia, it was essential to know the epidemiology of PR. Also, the study contributes to fill the gap between the national awareness and health care providers and give high quality of preventive and medical care.

\section{Objectives}

It was a situational analysis study designed to describe epidemiology and the burden of self-reported PR among pregnant Saudi women. Also, it evaluates the effect of medical and environmental factors on the prevalence of PR in Saudi Arabia. In addition to that, it identifies progression of rhinitis during pregnancy.

\section{Methods}

This was a biphasic multi-center prospective cross-sectional study. Sample size recommended was 228 with 6.5 margin of error, $95 \%$ confidence level and $50 \%$ response distribution based on Raosoft sample size calculator. Our target sample was 260.

The data were obtained from Saudi pregnant women attending the antenatal Clinics during June and July 2014. Random sampling was achieved by choosing one and skipping every five patients in the clinic list. The targeted areas of study were the largest and most popular eight governmental and private hospitals in Riyadh. They were King Abdulaziz Medical City, Maternity Hospital of Ministry of health; Military hospital; King Khalid University hospital; King Abdulaziz University hospital; Security forces Hospital, Al-Habeeb and Dallah hospital. These hospitals provide services to patients from different socio-economic classes and educational levels whom living in central region.

Subjects included in this research were all Saudi pregnant women in Riyadh following up in the mentioned 8 hospitals. Non-pregnant and non-Saudi women were excluded. The data were gathered using an Arabic language self-administered questionnaire that was validated in the pilot phase. During that, interviewers conducted the same questionnaire to be filled up among selected cases. Through the data collection, all missing data were recognized by the interviewers at the time of questionnaire submission and encouraged to be filled to grantee higher completeness rate. The study questionnaire involve three parts to collect data on personal and medical profile, pre-existing or newly appearing nasal symptoms with respect of pregnancy, information related to onset and remission of these symptoms, exaggerating factors, severity, environmental factors and smoking issues.

Our research added no harm to the patients and was according to Helsinki Declaration. It was approved by The King Abdullah International Medical Research Center (KAIMRC) and Institutional Review Board in NGHA, (IRP number SP14/103). All means of participant's right and respect were considered and implied consent to fill questionnaire was taken. Privacy and confidentiality of information were assured.

The data were coded and entered manually into white spreadsheets then transferred to SPSS statistical package version 16.0 for Windows. Standard quality assurance processes were implemented throughout all phases of data collection, coding, entry, cleaning, and analysis. Descriptive and comparative statistics were analyzed. Demographic data of study population were calculated in term of age and medical profile as descriptive analysis. The prevalence of self-reported of 
PR was calculated with $95 \%$ CI. Then cross tabulated analysis were performed for most of the variables to explore the multivariate association. The tests used were chi square (x2) test for determination of association between variables and $P$-value of $<0.05$ was considered significant. Fisher's Exact statistical test were used when Pearson correlation were violated. Appropriate tables and graphs were formed for description and simplification of results.

\section{Results}

The data were gathered from 247 out of 260 pregnant women with response and completeness rates of $95 \%$ and $99 \%$, respectively. Most of the participants lived in central region of Saudi Arabia. The reported rhinitis during pregnancy counted for $31.2 \%$ (CI 25.6\%-37.2\%).

The most frequent manifestations among PR cases were nasal pruritus $(67.5 \%)$, followed by sneezing (57.1\%), nasal congestion (50.6\%), and post nasal drip (46.7\%). The least reported manifestations were runny nose $(38.9 \%)$, coughing (35\%), and nasal obstruction (32.4\%). Other Ear, Nose or Throat symptoms including post nasal drainage, snoring, mouth breathing, lacrimation or itching of eyes, and associated smell and taste disorder were reported by $8.3 \%$ of participants who had rhinitis symptoms during pregnancy (Fig. 1). Nearly half of the participants (46.8\%) described their symptoms as a moderate degree. Mild cases were accounted for $35 \%$ of cases, while $18.2 \%$ were rated as severe.

Younger age group (below 30 years) had insignificant higher rate (51.9\%) among affected patient $(P<0.4)$ but preexisting allergic diseases were reported as risk to develop rhinitis of pregnancy. More than $70 \%$ of patients who had allergic rhinitis or sinusitis reported rhinitis in their pregnancy. Eye allergy and Bronchial asthma patients reported PR in $62.5 \%$ and $55 \%$ of them, respectively. Also, hormonal diseases patients reported PR in 29.7\% (Table 1).

Despite $23 \%$ of the suffers does not know their triggers, dust was reported to be the most common for PR symptoms by more than $70 \%$ of participants with PR. Tobacco/shisha smoke and perfume were the 2nd most triggering factors which were reported as $38 \%$ and $36 \%$, respectively. Incense and flowers contact were reported as third most triggers in $31 \%$ and $27.3 \%$, respectively. Medications and food were referred as a

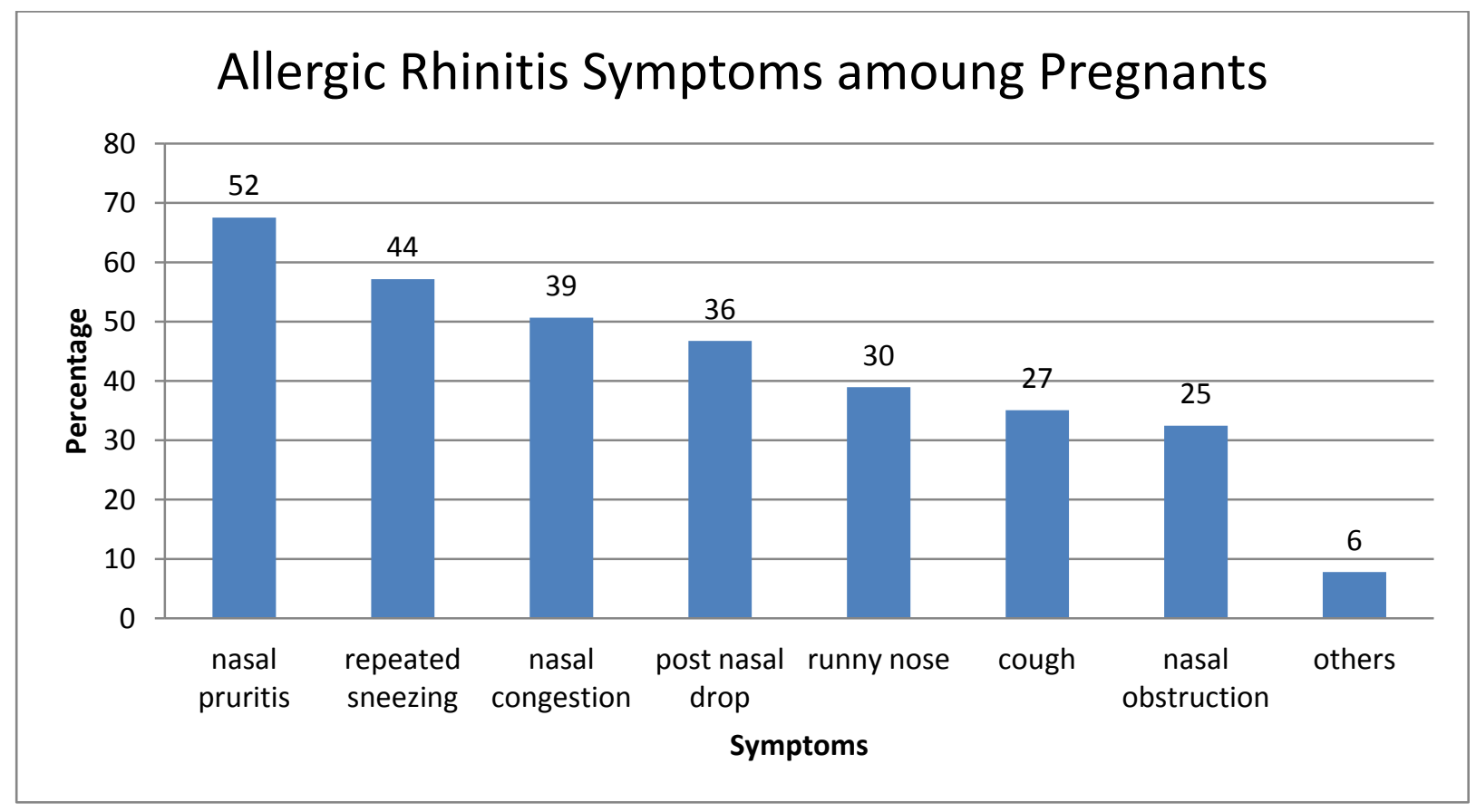

Fig. 1 The prevalence of rhinitis manifestation during pregnancy. 
Table 1 The prevalence of pregnancy rhinitis with respect to previous medical history.

\begin{tabular}{lllll}
\hline \multirow{2}{*}{ Medical history } & $*$ Total & $\mathrm{N}$ & New rhinitis manifestation during pregnancy \\
\cline { 2 - 5 } & $\mathrm{N}$ & 29 & $\%$ & $P$ value \\
\hline Allergic rhinitis & 40 & 11 & $72.5 \%$ & 0.000 \\
Hormonal disorders & 37 & 16 & $29.7 \%$ & 0.8 \\
Sinusitis & 22 & 11 & $72.7 \%$ & 0.000 \\
Bronchial Asthma & 20 & 5 & $55.0 \%$ & 0.01 \\
Eye Allergy & 8 & & $62.5 \%$ & 0.06 \\
\hline
\end{tabular}

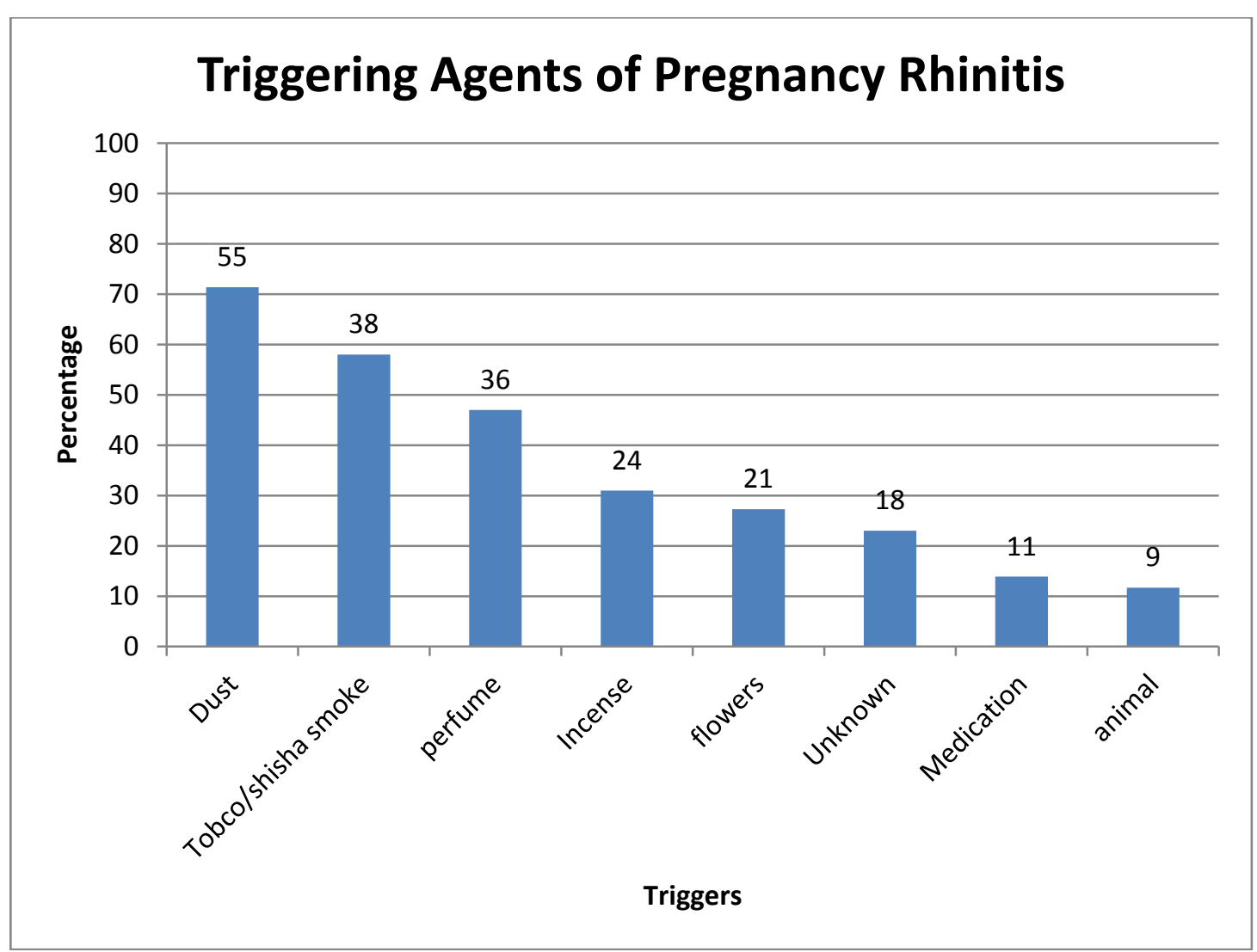

Fig. 2 The triggering agents of Pregnancy Rhinitis manifestations.

Table 2 Prognosis of PR symptoms with respect to the trimester.

\begin{tabular}{|c|c|c|c|c|c|c|c|c|c|c|}
\hline \multirow{3}{*}{$\begin{array}{l}\text { PR symptoms } \\
\text { started }\end{array}$} & \multicolumn{4}{|c|}{ No change } & \multicolumn{4}{|c|}{ Pregnancy Rhinitis symptoms worsen } & \multicolumn{2}{|r|}{ Total } \\
\hline & & & & imester & & 2nd Trimester & & 3rd Trimester & & \\
\hline & $\overline{\mathrm{N}}$ & $\%$ & $\mathrm{~N}$ & $\%$ & $\mathrm{~N}$ & $\%$ & $\mathrm{~N}$ & $\%$ & $\mathrm{~N}$ & $\%$ \\
\hline 1st trimester & 23 & $38.5 \%$ & 31 & $50.8 \%$ & 2 & $3.1 \%$ & 5 & $7.7 \%$ & 61 & $79.2 \%$ \\
\hline 2nd trimester & 5 & $38.5 \%$ & 0 & $0.0 \%$ & 2 & $23.1 \%$ & 5 & $38.5 \%$ & 12 & $15.6 \%$ \\
\hline 3rd trimester & 1 & $25.0 \%$ & 0 & $0.0 \%$ & 0 & $0.0 \%$ & 3 & $75.0 \%$ & 4 & $5.2 \%$ \\
\hline
\end{tabular}

triggering factor for PR in $13.9 \%$ of participants. Animals were the least reported aggravating factor (11.7\%) for PR (Fig. 2).

The prognosis of rhinitis symptoms during pregnancy was variable. As reported, majority of cases $(62.3 \%)$ get worsen but it depends on the trimester in which the symptoms started to appear. The majority (79.2\%) of PR manifestation started at the 1st trimester. About $50.8 \%$ of them got worsen during it and $7.7 \%$ got worse in the 3rd trimester. However $38.5 \%$ remained with no change. Among $15.6 \%$ of PR symptoms which started at the 2 nd trimester, $38.5 \%$ of 
cases worsened in 3rd trimester and a similar percentage remained unchanged. Also, seventy fifth percent of the of PR symptoms which started at the 3rd trimester get worsen. Most of the preexisting allergic conditions including rhinitis, sinusitis, bronchial asthma and eye allergy were shown to be either getting worse $(47 \%)$ or doesn't change (30.8\%) during pregnancy except $22 \%$ of them was improved (Table 2).

\section{Discussion}

Up to our knowledge, our study was the first reported multicenter study which analyzed the prevalence of PR in Saudi Arabia. Surprisingly, in many countries alike Saudi Arabia, the epidemiology of PR remains understudied. Based on many dynamics such as genetic and environmental factors, the prevalence rate of PR varies across countries and even within one country [4]. In our study, PR was estimated to be $31.2 \%$ among Saudi pregnant women. Consistently, PR has been estimated to be ranged between $18 \%$ in the United States and 39\% in Poland [7].

Despite PR remains unclear whether it is started due to pregnancy and the physiological changes, or due to aggravation of underling existing condition prior to pregnancy, several studies have addressed certain associations between demographic features and pregnancy rhinitis such as age and living area. Age group has no significant relation with PR in our study. Also, humid regions were shown in Wang study to cause sensitive reactions but this is not coherent with our study where the majority was living in the dry weather of central region [17].

Storaas and his colleagues recognized allergic family history, especially rhinitis, and environmental influences as risk factors for rhinitis [18]. In our study, many stimulants were agreed upon by participants to trigger rhinitis. Certain reported stimulants were significantly associated with new rhinitis symptoms during pregnancy which is coherent with Wallace study [1]. The majority (71.4\%) of PR sufferers reported dust as a triggering factor for rhinitis symptoms. The sandy weather of Riyadh might have an influenced that. Tobacco and shisha smoke were the second most aggravating factors for PR symptoms accounting for $58 \%$ which consistent with other studies [18]. Perfume, incense and flowers reported as trigger that induce rhinitis in pregnancy. Medications and food were referred as a triggering factor for PR in $13.9 \%$ of participants. It is believed that rhinitis symptoms can be triggered be certain foods, and medications such as aspirin, beta blockers, and nasal decongestion spray overuse [3]. Animals were the least common aggravating factor (11.7\%) for PR in this study. Although, sensitivity vulnerable individuals are at risk of developing symptoms when they are exposed to allergens of cats or dogs [1]. This may refer to cautiousness of pregnant women towards home pets. Around $23 \%$ of triggers were unknown, in which the participants were not sure of the specific factor causing the symptoms.

PR can develop anytime in pregnancy and disappears mostly within 2 weeks postpartum [7, 9]. In our study, the majority of participants (79.2\%) started complaining of PR symptoms at the first trimester. Almost half of the symptoms remained constant or had worsened at the trimester which appeared in, while others deteriorated at the 3rd trimester. These results were comparable to the West Pomerania study [7]. These changes are attributed to increased blood volume and hormonal alterations which are typically noticed in the first two trimesters, and have a direct influence on the nasal mucosa [10]. Symptoms started to decrease at the end of the pregnancy as blood volume begin to shifts to the extra vascular space. But PR is not fully obvious if it starts as a result of physiology related to pregnancy, or as an exacerbation of preexisting disorder. Deficient studies on rhinitis during pregnancy might have under evaluated the significance and process of PR and the factors influencing it.

There are well known health problems strongly 
associated with developing or deterioration of rhinitis, such as hypothyroidism [3]. Among hormonal disease patients, $29.7 \%$ of them developed rhinitis in their pregnancy which is coherent with Settipane study. Also, Bernstein and his colleagues stated that nearly $30 \%$ of woman with known allergic rhinitis are at risk of worsening of symptoms during pregnancy compared to $72 \%$ in our study [19]. That nominates former rhinitis as a strong prognosticator for developing symptoms during pregnancy. Co-existence of bronchial asthma and allergic rhinitis is well noticed [20].

\section{Recommendations}

Finally, we recommend that PR symptoms should be taken in consideration in pregnancy follow ups. This will have a great relief of the burden of these bothersome symptoms on pregnant women. We suggest further research with a large population-based study of PR in all regions of Saudi Arabia with more emphasis on the PR subtypes, risk factors, clinical history and examination.

\section{Conclusion}

Pregnancy rhinitis among Saudi pregnant women is common as $31.2 \%$ in which symptoms emerging mostly in the first trimester and gets worsen. Preexisting allergic diseases and exposure to aggravating factors were greatly associated with developing PR symptoms.

\section{Limitations}

We consider some limitations in our study. The sample may not be representative for all Saudi pregnant women or specific group of women, such as smokers or exposure to workplace hazards. Our findings might be over or underrated, since it was only based on the self-reported approach, instead of clinical history, examination, and related investigations.

\section{Acknowledgment}

We would like to recognize of KIMARC assistance for our study. Also, gynecology departments in the eight hospitals are appreciated.

\section{References}

[1] Wallace, D., Dykewicz, M., Bernstein, D., Blessingmoore, J., Cox, L., Khan, D., Lang, D., Nicklas, R., Oppenheimer, J., and Portnoy, J. 2008. "The Diagnosis and Management of Rhinitis: An Updated Practice Parameter." Journal of Allergy and Clinical Immunology 122 (2): S1-84.

[2] The American Academy of Allergy, Asthma \& Immunology. 2016. RHINITIS (HAY FEVER). Accessed June 20, 2016. http://www.aaaai.org/conditions-and-treatments/allergies/ rhinitis.

[3] Settipane, R. A., and Charnock, D. R. 2007. "Epidemiology of Rhinitis: Allergic and Nonallergic.' Clinical Allergy and Immunology 19: 23-34.

[4] Abdulrahman, H., Hadi, U., Tarraf, H., Gharagozlou, M., Kamel, M., Soliman, A., Hamad, W. A., Hanna, K. M., Mostafa, B. E., Omrani, M., Abdelmotal, A., and Moukarzel, N. 2012. "Nasal Allergies in the Middle Eastern Population: Results from the "Allergies in Middle East Survey"." American Journal of Rhinology and Allergy 26 (Suppl 1): S3-23.

[5] Sabry, E. Y. 2011. "Prevalence of Allergic Diseases in A Sample of Taif Citizens Assessed by An Original Arabic Questionnaire (Phase I)." Allergologia Et Immunopathologia 39 (2): 96-105.

[6] Loock, J.W. 2009. "Allergic Rhinitis and Pregnancy-A Review of the Literature, with Recommendations for Management." Current Allergy \& Clinical Immunology 22 (1): 11 .

[7] Zacharisen, M. C. 2000. "Rhinitis in Children, Adolescents, the Elderly, and Pregnant Women." Immunology and Allergy Clinics of North America 20 (2): 425-44.

[8] Ellegård, E. K. 2004. "Clinical and Pathogenetic Characteristics of Pregnancy Rhinitis." Clinical Reviews in Allergy \& Immunology 26 (3): 149-60.

[9] Dykewicz, M. S., and Daniel, L. H. 2010. "Rhinitis and Sinusitis." Journal of Allergy and Clinical Immunology 125 (2): S103-15.

[10] Goldstein, G., and Satish, G. 2012. "Rhinologic Issues in Pregnancy." Allergy \& Rhinology 3 (1): 13-5.

[11] Greiner, A. N., Peter, W. H., Guiseppina, R., and Glenis, K. S. 2011. "Allergic Rhinitis." The Lancet 378 (9809): 2112-22.

[12] Incaudo, G. A., and Patricia, T. 2006. "The Diagnosis and Treatment of Allergic Rhinitis during Pregnancy and Lactation." Immunology and Allergy Clinics of North America 26 (1): 137-54. 
[13] Piette, V., Jean-Pierre, D., and Pascal, D. 2012. "Erratum to: Treating Allergic Rhinitis in Pregnancy." Current Allergy and Asthma Reports 12 (3): 261.

[14] Incaudo, G. A. 2004. "Diagnosis and Treatment of Allergic Rhinitis and Sinusitis during Pregnancy and Lactation." Clinical Reviews in Allergy \& Immunology 27 (2): 159-78.

[15] Pregnancy-Info.Net. 2016. Pregnancy Symptoms and Discomfort: Rhinitis during Pregnancy. Accessed June 20, 2016. http://www.pregnancy-info.net/rhinitis.html.

[16] Wheeler, P. W., and Wheeler, S. F. 2005. "Vasomotor Rhinitis.” Am. Fam. Physician 72 (6): 1057-62.

[17] Wang, D. Y. 2005. "Risk Factors of Allergic Rhinitis: Genetic or Environmental?" Therapeutics and Clinical Risk Management 1 (2): 115-23.

[18] Storaas, T., Steinsvåg, S. K., Florvaag, E., Irgens, A., and Aasen, T. B. 2005. "Occupational Rhinitis: Diagnostic
Criteria, Relation to Lower Airway Symptoms and IgE Sensitization in Bakery Workers." Acta Oto-Laryngologica 125 (11): 1211-7.

[19] Bernstein, I. L., Li, J. T., Bernstein, D. I., Hamilton, R., Spector, S. L., Tan, R., Sicherer, S., Golden, D. B., Khan, D. A., Nicklas, R. A., Portnoy, J. M., Blessing-Moore, J., Cox, L., Lang, D. M., Oppenheimer, J., Randolph, C. C., Schuller, D. E., Tilles, S. A., Wallace, D. V., Levetin, E., Weber, R.; American Academy of Allergy, Asthma and Immunology; American College of Allergy, Asthma and Immunology. 2008. "Allergy Diagnostic Testing: An Updated Practice Parameter." Annals of Allergy, Asthma \& Immunology 100 (3): S1-148.

[20] Mandhane, S. N., Jigar, H. S., and Rajamannar, T. 2011. "Allergic Rhinitis: An Update On Disease, Present Treatments And Future Prospects." International Immunopharmacology 11 (11): 1646-62. 